\title{
Tunable slow- and fast-light devices based on molecular-aggregate nanofilms
}

\author{
E. Díaz, ${ }^{1}$ A. Villas, ${ }^{1}$ E. Cabrera-Granado,${ }^{2}$ and O. G. Calderón ${ }^{2}$ \\ ${ }^{1}$ GISC, Departamento de Física de Materiales, Universidad Complutense, E-28040 Madrid, Spain \\ ${ }^{2}$ Departamento de Óptica, Universidad Complutense, E-28037 Madrid, Spain
}

(Received 7 June 2018; published 9 October 2018)

\begin{abstract}
We study the tunability of nanofilms composed of linear molecular aggregates for slow- and fast-light performance. In order to describe a wide range of intensity field regimes, we consider a two-exciton model for the molecular aggregate where exciton creation or annihilation may occur. Our simulations show fractional delays and advancements of the order of those found in longer propagating media. This nanometric device presents two functionalities: (i) it allows the existence of slow or fast light in a different bandwidth of pulsed signals and (ii) such dual performance can be tuned by a small perturbation of the input signal amplitude for a fixed pulse temporal width. Both effects are tested under the usual presence of disorder in these molecular systems. This study concludes that a molecular-aggregate nanofilm presents a wide control of group velocity in the $\mathrm{GHz}$ and $\mathrm{THz}$ bandwidths.
\end{abstract}

DOI: 10.1103/PhysRevA.98.043818

\section{INTRODUCTION}

Control of the speed of light down to nanometer scale is a crucial challenge in the development of slow- and fast-light compact devices for all-optical information processing, especially when combining wide bandwidths reaching gigahertz $(\mathrm{GHz})$ or terahertz $(\mathrm{THz})$ frequencies and low losses.

Recent research has tried to achieve these goals. Mork et al. [1] showed group velocities of $\sim c / 100$ in a $100 \mu \mathrm{m}$ semiconductor waveguide at $\mathrm{GHz}$ frequencies. Similarly, micrometric composites doped with metal nanoparticles were proposed in [2] to obtain temporal delays up to 40 times the initial temporal pulse width in the $\mathrm{THz}$ range. Further reduction of the device dimensions down to the nanometer scale could be achieved by $J$-aggregate nanofilms based on the slow-light process of coherent population oscillations (CPO) [3]. In such a process, a time-periodic modulation of the population of an excited level is induced by the interaction with a strong control field and a probe field with slightly different frequency. This creates a narrow hole in the absorption spectrum whose linewidth is proportional to the inverse of the relaxation lifetime of the excited level. Operating as a slow-light device, a film of some tens of nanometers thickness composed of $J$-aggregates has shown to provide delays up to 0.5 times the pulse length for $\sim 14 \mathrm{GHz}$ bandwidth in the absence of size dispersion. Moreover, the probe pulse shows a gain, thus overcoming the usual insertion losses of slow-light devices. These figures of merit are robust against the presence of disorder and two-exciton dynamics which is relevant for high-intensity fields [4]. For a further review on $J$-aggregate optical properties, see Refs. [5,6].

To reach the fast-light regime, the common approach is to generate population inversion in the material [7]. Then, the $\mathrm{CPO}$-induced hole in the gain profile translates into a region of anomalous dispersion, and thus, into a temporal advancement of the propagating signal. However, this mechanism needs an additional external pump to ensure signal amplification, and the usable bandwidth is limited by the population response time [8]. Larger bandwidths can be accessible by the anomalous dispersion region associated with the natural resonance of atomic systems. Still, significant time advancement is always accompanied, through Kramers-Kronig relations, by strong attenuation, thus making it difficult to measure. To avoid this problem, several artificial processing methods such as gain-assisted linear anomalous dispersion [8] have been used to demonstrate superluminal-light propagation. Nevertheless, few experiments have been able to achieve fast-light propagation using the linear anomalous dispersion of a single absorption line. For example, Chu and Wong [9] obtained group velocities of $-10^{8} \mathrm{~cm} / \mathrm{s}$ in a tens of micron-sized GaP:N sample, whereas Tanaka et al. [10] observed a fractional advancement of $25 \%$ in a $1-\mathrm{cm}$-thick $\mathrm{Rb}$ vapor cell. Samples with sizes comparable to or smaller than the wavelength of the excitation light have been used to decrease the strong attenuation, since it varies with sample size more rapidly than the advance. For example, Keaveney et al. [11] observed over 100 ps advance across a 390-nm-thick dense atomic vapor. More recently, Jennewein et al. [12] showed delays as large as $-10 \mathrm{~ns}$, corresponding to negative group velocities $\sim-300 \mathrm{~m} / \mathrm{s}$, in a dense cloud of cold atoms. In this line, the subwavelength film thickness and the high oscillator strength of $J$-aggregate nanofilms make them good candidates to observe fast-light propagation in the linear absorption regime, where $\mathrm{THz}$ bandwidths can be achieved with no need of an external pump.

Here we analyze the performance of a $J$-aggregate nanofilm as a tunable slow- and fast-light device using the two-exciton model already employed in previous works [4]. As stated before, we combine CPO-based slow light with fast light provided by the anomalous dispersion naturally present in the aggregate absorption linewidth. We show how the coherent response of these aggregates allows us to reach significant advancements with constrained absorption for bandwidths up to the $\mathrm{THz}$ range. Moreover, it can overcome 
the strong attenuation occurring in other fast-light devices, therefore, removing the need of additional pumping. We then analyze the optimum conditions to tune the temporal shift of modulated signals from delay to advancement. First, we numerically study a four-level model where excitonic states with 0,1 , or 2 excitons, and exciton-exciton annihilation by way of vibrational states is also considered. In addition, since the latter is clearly faster than the decay rates of the rest of states, we simplify the system to a three-level model and apply a perturbation treatment which allows us to obtain some analytical results. Last, we analyze a one-exciton approximation in order to get deeper insight into the physical processes responsible for the main features of slow- and fast-light propagation. We must stress that the one-exciton model accounts for a different physical system with respect to the original one, but it allows us to obtain closed analytical expressions for the main magnitudes of interest.

\section{THEORETICAL FRAMEWORK}

As in previous studies [3,4], we consider an ultrathin film consistent of an ensemble of linear molecules oriented parallel to each other where exciton-exciton interaction plays a role. Exciton states are localized over a particular length $N$ of the aggregate due to the disorder that is usually present in these systems. Although light interaction with a $J$-aggregate is usually analyzed by the Frenkel exciton model, a complete treatment of the monomer optical dynamics and its interaction with neighbor molecules is untractable. To reduce the complexity of the system, we employ the approximate four-level model developed in Refs. [13-15]. This model is based on the following considerations. First, it describes the dynamics of a single localization segment with a length much shorter than the aggregate length. Second, it only contemplates the dominant transitions, carrying more than $70 \%$ of the oscillator strength, in both the one- and two-exciton manifolds. And last, intrasegment exciton-exciton annihilation is taken into account as a deexcitation route from the two-exciton level to the one-exciton or ground state through a molecular vibronic system. This model reproduces the pump-probe spectrum calculated without approximations [16], as well as the experimentally found blueshift of the one- to two-exciton transition [17]. Last, it has been already used to establish $J$-aggregate nanofilms as a promising platform for slow-light performance [4].

Figure 1 represents the schematics of the possible excitonic transitions of the model. $|0\rangle,|1\rangle$, and $|2\rangle$ refer to excitonic states with 0, 1, or 2 excitons. Exciton-exciton annihilation occurs by means of a set of electronic-vibrational levels (3) e-vib. $_{\text {. }}$ Such high-energy states relax to the ground vibrational state due to phonon-assisted processes, to finally be transferred again to $|0\rangle$ or $|1\rangle$.

Our formalism is based on the time-dependent density matrix described by way of Bloch equations under rotating wave and slowly varying amplitude approximations as follows:

$$
\begin{aligned}
\dot{\rho}_{00}^{N}= & i d_{10}^{N}\left(\sigma_{10}^{N} E^{*}-\sigma_{10}^{N *} E\right) / 4 \hbar+\gamma_{10}^{N} \rho_{11}^{N}+\gamma_{30}^{N} \rho_{33}^{N}, \\
\dot{\rho}_{11}^{N}= & i d_{21}^{N}\left(\sigma_{21}^{N} E^{*}-\sigma_{21}^{N *} E\right) / 4 \hbar-i d_{10}^{N}\left(\sigma_{10}^{N} E^{*}-\sigma_{10}^{N *} E\right) / 4 \hbar \\
& -\gamma_{10}^{N} \rho_{11}^{N}+\gamma_{21}^{N} \rho_{22}^{N}+\gamma_{31}^{N} \rho_{33}^{N},
\end{aligned}
$$

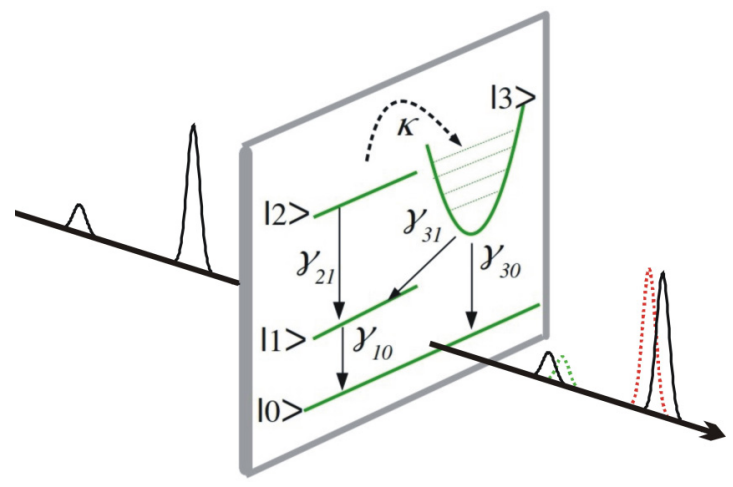

FIG. 1. Schematic view of the aggregate nanofilm modeled as an ensemble of four-level systems. Input signals (solid lines) of different intensity from left to right through the nanofilm results in an advanced (green) or delayed (red) output signal (dashed line).

$$
\begin{aligned}
\dot{\rho}_{22}^{N}= & -i d_{21}^{N}\left(\sigma_{21}^{N} E^{*}-\sigma_{21}^{N *} E\right) / 4 \hbar-\left(\gamma_{21}^{N}+\kappa\right) \rho_{22}^{N}, \\
\dot{\rho}_{33}^{N}= & -\left(\gamma_{30}^{N}+\gamma_{31}^{N}\right) \rho_{33}^{N}+\kappa \rho_{22}^{N}, \\
\dot{\sigma}_{10}^{N}= & {\left[i\left(\omega-\omega_{10}^{N}\right)-\Gamma_{10}^{N}\right] \sigma_{10}^{N} } \\
& -i d_{10}^{N}\left(\rho_{11}^{N}-\rho_{00}^{N}\right) E / \hbar+i d_{21}^{N} \sigma_{20}^{N} E^{*} / 2 \hbar, \\
\dot{\sigma}_{21}^{N}= & {\left[i\left(\omega-\omega_{21}^{N}\right)-\Gamma_{21}^{N}-\kappa / 2\right] \sigma_{21}^{N} } \\
& -i d_{21}^{N}\left(\rho_{22}^{N}-\rho_{11}^{N}\right) E / \hbar-i d_{10}^{N} \sigma_{20}^{N} E^{*} / 2 \hbar, \\
\dot{\sigma}_{20}^{N}= & {\left[i\left(\omega-\omega_{10}^{N}\right)+i\left(\omega-\omega_{21}^{N}\right)-\Gamma_{20}^{N}-\kappa / 2\right] \sigma_{20}^{N} } \\
& +i d_{21}^{N} \sigma_{10}^{N} E / 2 \hbar-i d_{10}^{N} \sigma_{21}^{N} E / 2 \hbar .
\end{aligned}
$$

Here $E$ refers to the slowly varying amplitude of the field whose frequency is $\omega$. The remaining magnitudes, as indicated by the superscript, will depend in general on the localization length of every coherent segment $N$. The diagonal element $\rho_{j j}^{N}$ denotes the population of the energy level $j$, while $\sigma_{i j}^{N}$ is the slowly varying amplitude of the coherence between the energy levels $(i, j)$. The transition frequency and the dipole moment within those levels of every segment read $\omega_{i j}^{N}$ and $d_{i j}^{N}=d_{i j}^{1} \sqrt{N}$ respectively. Hereafter the superscript 1 refers to properties of a monomer. Spontaneous emission rates are $\gamma_{i j}^{N}$ while $\Gamma_{i j}^{N}$ are the decay of the coherences. In particular, $\Gamma_{10}^{N}=\gamma_{10}^{N} / 2+\Gamma, \Gamma_{21}^{N}=\gamma_{10}^{N} / 2+\gamma_{21}^{N} / 2+\Gamma$, and $\Gamma_{20}^{N}=\gamma_{21}^{N} / 2+\Gamma$, where $\Gamma$ accounts for pure nonradiative processes. The relaxation rates of the vibronic state $(3)_{\mathrm{e} \text {-vib }}$ to $|0\rangle$ and $|1\rangle$ are $\gamma_{30}^{N}$ and $\gamma_{31}^{N}$ respectively. Last, $\kappa$ refers to the exciton-exciton annihilation. According to Ref. [18], size dispersion of the coherent segments turns into an inhomogeneous broadening affecting the $\mathrm{J}$ band, and therefore, it gives rise to the fluctuation of the transition energies $\hbar \omega_{10}^{N}$ and $\hbar \omega_{21}^{N}$. This effect is taken into account in our model by substituting all the size-dependent quantities, except for $\omega_{10}^{N}$ and $\omega_{21}^{N}$, by their mean values in the aggregate.

To maintain the crucial physics of the model while keeping the mathematics treatable, we contemplate the following concerns $[3,4]$. On the one hand we explicitly keep the dimensional magnitudes of all transition rates to clearly provide the signal bandwidth accessible by the slow- and fast-light device. This allows us to make a direct comparison with other 
experimental slow- and fast-light setups. On the other hand, however, the input signal amplitude is taken into account by means of its associated dimensionless Rabi frequency. This approach is quite standard when analyzing the interaction of light with atomic or molecular systems. For brevity, we will refer to the field by way of the Rabi frequency defined in units of $\Gamma_{10}$ as $\Omega=d E / \hbar \Gamma_{10}$ from now on, where $d=\sqrt{\left(d_{10}^{2}+d_{21}^{2}\right) / 2}$. This normalization choice guarantees that the most relevant results of our study will arise within the range $|\Omega| \in[0-10]$ and thus, it simplifies the numerical analysis. Hereafter we assume normal incidence and parallel polarization of the field to the transition dipole moments of all the aggregates and to the film plane. The field inside the film results from two contributions: the external field $\Omega^{\text {in }}$ and the electric polarization of the disordered molecular aggregates. Thus, it can be written as $\Omega=\Omega^{\text {in }}+i \gamma_{R} \sum_{N} p(N)\left(\mu_{10} \sigma_{10}+\right.$ $\left.\mu_{21} \sigma_{21}\right)$, where $\mu_{i j}=d_{i j} / d$ and $p(N)$ refers to the disorder distribution over localization lengths. The collective superradiant damping of the aggregate is $\gamma_{R}=\mu_{0}|d|^{2} N_{0} c \omega L / 2 \hbar \Gamma_{10}$, where $N_{0}$ is the density of localization segments, $L$ is the nanofilm thickness, and $\mu_{0}$ is the vacuum magnetic permeability $[19,20]$.

In our study we integrate Eqs. (1) for every coherent segment to calculate its contribution to the electric polarization, such that the total molecular field results from the following average: $\sum_{N} p(N) \propto \int_{-\infty}^{\infty} \exp \left[\frac{-\left(\Delta_{10}^{\wedge}-\Delta_{10}\right)^{2}}{2 G^{2}}\right] d \Delta_{10}^{N}$. Here, $G$ denotes the inhomogeneous broadening of the J bandwidth in units of $\Gamma_{10}$, and $\Delta_{10}$ refers to the detuning with respect to the central frequency of the Gaussian. For simplicity we consider an incident resonant field such as $\omega=\omega_{10}$.

In this paper we will study the response of the system to a general modulated signal $\Omega=\Omega_{0}+\Omega_{m}(t)$, such that the transmittance $T$ and the fractional shift $F$ induced by the film is calculated by the ratio between the output and input signals as

$$
\Omega_{m} / \Omega_{m}^{\text {in }}=T \exp (i 2 \pi F) .
$$

Notice that $T$ and $F$ will be evaluated by means of the modulus and the complex argument of the complex ratio $\Omega_{m} / \Omega_{m}^{\text {in }}$, respectively. The fractional shift can also be expressed in terms of the temporal shift of the outgoing signal with respect to the incident one, $t_{s}$. In such a case $F=t_{s} / T_{0}$, where $T_{0}$ is the period of a sinusoidally modulated signal, or the pulse FWHM in the case of a pulsed signal.

\section{Three-level equivalent model}

Before analyzing the results provided by numerical integration of Eqs. (1) let us develop a simplified three-level model that retains the main relevant features of the complete set of equations but allows us to obtain analytical results. We will focus on a homogeneous aggregate nanofilm, i.e., neglecting the dispersion over localization lengths $N$. Thus, we will suppress the superindex $N$ in all variables. The role of the disorder distribution will be discussed later. First, we notice that $\rho_{22} \simeq 0$ can be established as exciton-exciton annihilation, which rapidly transfers the energy from the two-exciton state to the electronic-vibrational levels. Moreover, we can neglect $\sigma_{20}$, i.e., the coherence between the two-exciton level and the ground state. Last, we consider that the incident field frequency nearly matches the frequencies of the two relevant transitions. Then, the equations governing the dynamics of a homogeneous aggregate system remain:

$$
\begin{aligned}
\dot{\rho}_{00}= & \gamma_{10} \rho_{11}+\gamma_{30} \rho_{33}+i \Gamma_{10} \mu_{10}\left(\sigma_{10} \Omega^{*}-\sigma_{10}^{*} \Omega\right) / 4, \\
\dot{\rho}_{11}= & -\gamma_{10} \rho_{11}+\gamma_{31} \rho_{33}+i \Gamma_{10} \mu_{21}\left(\sigma_{21} \Omega^{*}-\sigma_{21}^{*} \Omega\right) / 4 \\
& -i \Gamma_{10} \mu_{10}\left(\sigma_{10} \Omega^{*}-\sigma_{10}^{*} \Omega\right) / 4, \\
\dot{\rho}_{33}= & -i \Gamma_{10} \mu_{21}\left(\sigma_{21} \Omega^{*}-\sigma_{21}^{*} \Omega\right) / 4-\left(\gamma_{30}+\gamma_{31}\right) \rho_{33}, \\
\dot{\sigma}_{10}= & -\Gamma_{10} \sigma_{10}-i \Gamma_{10} \mu_{10}\left(\rho_{11}-\rho_{00}\right) \Omega, \\
\dot{\sigma}_{21}= & -\left(\Gamma_{21}+\kappa / 2\right) \sigma_{21}+i \Gamma_{10} \mu_{21} \rho_{11} \Omega .
\end{aligned}
$$

In this work, we use a broad range of temporal pulse widths reaching values larger than the dephasing times, and therefore the coherences $\sigma_{10}$ and $\sigma_{21}$ cannot be adiabatically eliminated, contrary to the common situation in CPO-based slow-light studies [21]. Notice that all these initial assumptions will be fully validated in the next section when we compare the numerical results from the complete [Eqs. (1)] and the reduced [Eqs. (4)] models. We study the response of the $J$-aggregate nanofilm to a field $\Omega=\Omega_{0}+$ $\Omega_{m} \exp (-i \delta t)+$ c.c., with a strong control field $\Omega_{0}$ at frequency $\omega$ and two sidebands $\Omega_{m}$ at frequencies $\omega \pm \delta$. This field induces a periodic modulation of the populations and the coherences at $\delta, \rho_{j j}=\rho_{j j 0}+\rho_{j j m} \exp (-i \delta t)+$ c.c. and $\sigma_{i j}=\sigma_{i j 0}+\sigma_{i j m} \exp (-i \delta t)+$ c.c.. We insert this expansion into the simplified Eqs. (3) and equate terms oscillating at the same harmonic of $\delta$ [22]. The 0 order, related to the strong field, corresponds to the steady-state case. The first-order term in $\delta$ gives the amplitude of the population oscillation and the sidebands fields.

$$
\begin{aligned}
\rho_{110}= & \frac{\mu_{10}^{2} \Omega_{0}^{2} / 2}{\frac{\gamma_{10}}{\Gamma_{10}}+\mu_{10}^{2} \Omega_{0}^{2}+\frac{\Gamma_{10} \mu_{21}^{2} \Omega_{0}^{2}}{2\left(\Gamma_{21}+\kappa / 2\right)}\left(1-\frac{\gamma_{31}}{\gamma_{3}}+\frac{\Gamma_{10} \mu_{10}^{2} \Omega_{0}^{2}}{2 \gamma_{3}}\right)}, \\
\rho_{330}= & \frac{\mu_{21}^{2} \Omega_{0}^{2} \Gamma_{10}^{2}}{2 \gamma_{3}\left(\Gamma_{21}+\kappa / 2\right)} \rho_{110}, \\
\frac{\Omega_{0}^{\text {in }}}{\Omega_{0}}= & +\gamma_{R} \mu_{10}^{2}\left(1-\rho_{330}-2 \rho_{110}\right)+\frac{\gamma_{R} \rho_{110} \mu_{21}^{2} \Gamma_{10}}{\Gamma_{21}+\kappa / 2}, \\
\frac{\rho_{11 m}}{\Omega_{m}}= & -\frac{\Omega_{0}}{2}\left[\mu_{10}^{2}\left(2 \rho_{110}+\rho_{330}-1\right) D_{10}+\mu_{21}^{2} \rho_{110} D_{21}\right. \\
& \left.\times\left(1-\frac{\gamma_{31}}{F_{3}}+\frac{\mu_{10}^{2} \Omega_{0}^{2} \Gamma_{10}^{2}}{2 F_{10} F_{3}}\right)\right] /\left[\frac{\gamma_{10}}{\Gamma_{10}}-i \frac{\delta}{\Gamma_{10}}\right. \\
& \left.+\frac{\mu_{10}^{2} \Omega_{0}^{2} \Gamma_{10}}{F_{10}}+\frac{\mu_{21}^{2} \Omega_{0}^{2} \Gamma_{10}}{2 F_{21}}\left(1-\frac{\gamma_{31}}{F_{3}}+\frac{\mu_{10}^{2} \Omega_{0}^{2} \Gamma_{10}^{2}}{2 F_{10} F_{3}}\right)\right], \\
\frac{\Omega_{m}^{\text {in }}}{\Omega_{m}}= & 1-\gamma_{R} \Omega_{0} \Gamma_{10} \frac{\rho_{11 m}}{\Omega_{m}}\left[\frac{2 \mu_{10}^{2}}{F_{10}}-\frac{\mu_{21}^{2}}{F_{21}}+\frac{\mu_{10}^{2} \mu_{21}^{2} \Omega_{0}^{2} \Gamma_{10}^{2}}{2 F_{10} F_{3} F_{21}}\right] \\
& -\gamma_{R} \Gamma_{10}\left[\frac{\mu_{10}^{2}}{F_{10}}\left(2 \rho_{110}+\rho_{330}-1\right)-\frac{\mu_{21}^{2}}{F_{21}} \rho_{110}\right. \\
& \left.+\frac{\mu_{10}^{2} \mu_{21}^{2} \Omega_{0}^{2} \Gamma_{10}}{2 F_{10} F_{3}} D_{21} \rho_{110}\right],
\end{aligned}
$$

where $F_{10}=\Gamma_{10}-i \delta, \quad F_{21}=\Gamma_{21}+\kappa / 2-i \delta, \quad$ and $F_{3}=$ $\gamma_{3}-i \delta . D_{10}=1+\Gamma_{10} / F_{10}$ and $D_{21}=\Gamma_{10} /\left(\Gamma_{21}+\kappa / 2\right)+$ $\Gamma_{10} / F_{21}$. The last equation (4) gives us the transmission and 

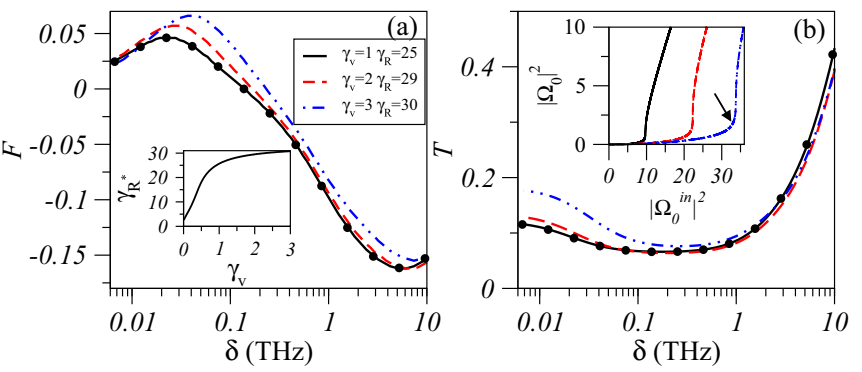

FIG. 2. Fractional shift (a) and transmittance (b) of a sinusoidal input signal as a function of the frequency $\delta$ for different values of $\gamma_{v}$ and $\Omega_{0}^{\text {in }}=\Omega_{0}^{\text {sat }} / 2$ in absence of disorder. Inset (a) shows the optical bistability threshold $\gamma_{R}^{*}$, estimated by way of the equivalent model (4), as a function of the parameter $\gamma_{v}$. Inset (b) presents the output-input curve for the strong field intensity $\left|\Omega_{0}\right|^{2}$, with the arrow pointing to the saturation intensity for $\gamma_{v}=3$. Symbols result from analytical calculations based on Eqs. (4).

delay time suffered by the sideband. The second term of the right-hand side of this equation, which is proportional to $\rho_{11 \mathrm{~m}}$, is produced by the CPO mechanism, leading to slow light. The last term of the right-hand of this equation is the usual propagation in the anomalous dispersion region of a natural atomic transition, leading to fast light.

\section{FIGURES OF MERIT FOR SLOW AND FAST LIGHT}

\section{A. Homogenous aggregate nanofilm}

As a control scenario, we will start by studying the slow- and fast-light performance of the molecular-aggregate nanofilm when the incident signal is sinusoidally modulated, $\Omega=\Omega_{0}+\Omega_{m} \sin (\delta t)$. This incident field will also test the previous simplified model developed in Eqs. (4). For the time being we will show our calculations for a homogeneous aggregate of size $N=100$ [23]. We use PIC-Br as a reference widely studied aggregate. As mentioned, we will work with incident fields such that $\omega=\omega_{10}$ while $\hbar\left(\omega_{21}-\right.$ $\left.\omega_{10}\right)=3 \pi^{2} J / N^{2}=1 \mathrm{meV}$ when the nearest-neighbor interaction is $J=80 \mathrm{meV}$. We use $\gamma_{10}^{1}=1 / 3.7 \mathrm{~ns}^{-1}, \gamma_{i j}=$ $\gamma_{10}^{1}\left|\mu_{i j}^{2}\right|$, and $\Gamma_{10}=\gamma_{10} / 0.02$. Finally, $\kappa=5000 \mathrm{~ns}^{-1}$. Notice that the exciton-exciton annihilation rate is the fastest process in the system. These values are consistent with measurements at low temperatures [24,25] and with the estimation of the exciton-exciton annihilation rate given by [14]. The transition dipole moments are $d_{10}^{1}=12.1 \mathrm{D}$ and $d_{21}=\sqrt{1.5} d_{10} \mathrm{D}$, and the concentration of aggregates is $N_{0} \sim 10^{23} \mathrm{~m}^{-3}$. Notice that we have considered the average ratio of the oscillator strength of the relevant transitions as $f_{21} / f_{10} \sim d_{21}^{2} / d_{10}^{2} \sim 1.5$ [25]. Last, though to the best of our knowledge there is no experimental measurement of the rates $\gamma_{31}$ and $\gamma_{30}$, the employed values in our study are based on those found in the bibliography [26]. According to our simulations, the slow- and fast-light performance is relevant when $\left(\gamma_{30}+\gamma_{31}\right) \gtrsim \gamma_{10}$ so for simplicity we take equal decay values $\gamma_{30}=\gamma_{31}=\gamma_{v} \gamma_{10}$ and we vary the ratio $\gamma_{v}$. Figure 2 depicts the fractional shift and transmittance, calculated by integration of Eqs. (1) as a function of the modulation frequency $\delta$ and different values
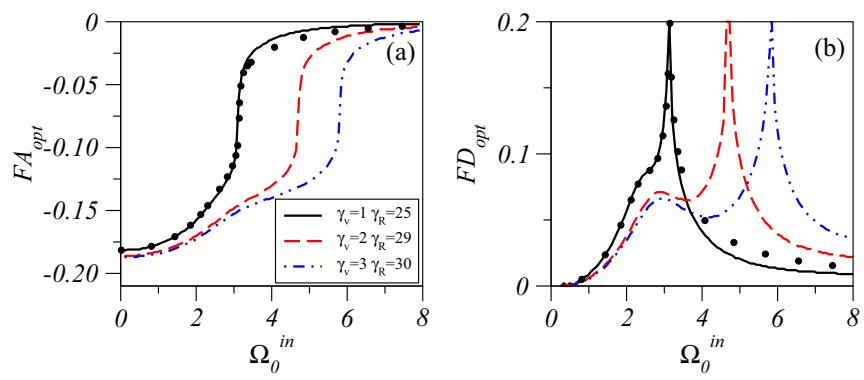

FIG. 3. (a) Maximum fractional advancement $F A_{\text {opt }}$ and (b) maximum fractional delay $F D_{\mathrm{opt}}$ as a function of the input strong field $\Omega_{0}^{\text {in }}$ for different values of the ratio $\gamma_{v}$ in absence of disorder. Symbols result from analytical calculations based on Eqs. (4).

of $\gamma_{v}$. Notice that negative (positive) values of $F$ are related to fast- (slow-)light processes.

Previous works have theoretically demonstrated the mirrorless bistable response of molecular-aggregate nanofilms [13,27-29]. In particular, for the one-exciton model, the nanofilm exhibits bistability if $\gamma_{R} \geqslant \gamma_{R}^{*}=8$. In the two-exciton model, the optical bistability threshold value, $\gamma_{R}^{*}$, depends on the full set of parameters of the four-level model and it can only be calculated numerically. However, this task is less complex for the equivalent model (4). Inset of Fig. 2(a) shows the parameter $\gamma_{R}^{*}$ as a function of $\gamma_{v}$. Such a value has been numerically estimated by searching the bistability condition in Eqs. (4), that is, by finding the value of $\gamma_{R}^{*}$ that gives rise to a multiple-evaluated solution of $\Omega_{0}$ as a function of $\Omega_{0}^{\text {in }}$.

In Refs. [3,4] the optimum value for the slow-light performance was demonstrated to appear if $\gamma_{R} \lesssim \gamma_{R}^{*}$ and $\Omega_{0}^{\text {in }}=$ $\Omega_{0}^{\text {sat }}$, where $\left|\Omega_{0}^{\text {sat }}\right|^{2}$ is the saturation intensity. This is the intensity for the critical point indicated by an arrow in the inset of Fig. 2(b) and it is associated with a critical incident field for which the reflection of the film drops abruptly in favor of transmission that rises up strongly. Such saturation intensity is well defined in a two-level system. For example, if we restrict ourselves to the transition from the $|0\rangle$ - to $|1\rangle$-exciton state it would read $\Omega_{0}^{\mathrm{sat}}(|0\rangle \rightarrow|1\rangle)=\sqrt{\gamma_{10} / \Gamma_{10}}$ [30]. However, in our four-level model, in order to saturate the complete system one must take into account not only the $|0\rangle \rightarrow|1\rangle$ but also the $|1\rangle \rightarrow|2\rangle$ transition and the decay through the electronicvibrational levels. As a result its expected value will be higher and its analytical evaluation is rather more complex. Thus, we compute this value analyzing the input-output intensity field curve, where an abrupt change of the output signal occurs, as pointed out in the inset of Fig. 2(b). When searching for fast-light performance, Fig. 3 demonstrates that the fractional advancement is larger for incident fields well below $\Omega_{0}^{\text {sat }}$. However, the condition $\gamma_{R} \lesssim \gamma_{R}^{*}$ is still valid. In order to propose a tunable device that can produce delay or advancement for a particular input signal as a function of the incident field, we will show results for $\Omega_{0}^{\text {in }}=\Omega_{0}^{\text {sat }} / 2$ and $\gamma_{R} \lesssim \gamma_{R}^{*}$ unless stated otherwise. Be aware that $\gamma_{R}^{*}$ depends on $\gamma_{v}$.

Figure 2(a) shows that signals with modulation frequency such that $\delta \in[5-500] \mathrm{GHz}(\delta \in[500 \mathrm{GHz}, 10 \mathrm{THz}])$ will be delayed (advanced) when propagating through the nanofilm. Indeed, for both regimes, there is a particular modulation 
frequency that shows a maximum fractional delay (advancement). Figure 2(b) shows an increasing transmission with larger $\delta$ as well. It is worth noting that for the optimal scenario for slow-light performance $\left(\Omega_{0}^{\text {in }}=\Omega_{0}^{\text {sat }}\right)$, there is gain in the weak sinusoidal modulation as shown in Ref. [3]. However, when the latter is not fulfilled or in the fast-light regime, the modulated signal gets weaker when propagating through the nanofilm, which is the usual scenario in longer propagating media. As shown, results are similar for different values of $\gamma_{v}$.

Let us analyze the optimum fractional advancement ( $\left.F A_{\text {opt }}\right)$ and delay ( $\left.F D_{\text {opt }}\right)$ as a function of the incident field, see Fig. 3. Such an optimum value will appear for a different modulation frequency for every field. Figure 3(a) shows that $\left|F A_{\text {opt }}\right|$ increases when $\Omega_{0}^{\text {in }}$ is weaker, while $F D_{\text {opt }}$ is maximum when $\Omega_{0}^{\text {in }}=\Omega_{0}^{\text {sat }}$. Indeed $\left|F A_{\text {opt }}\right|$ suffers an abrupt fall for the latter condition that maximizes $F D_{\text {opt }}$. In both Figs. 2 and 3, we show good agreement between numerical results obtained with the four-level model (1) and those analytically obtained with the simplified model (4) for $\gamma_{v}=1$.

For greater insight into the tunability of slow and fast light shown in our system, let us consider a further simplification in our model, i.e., the one-exciton approximation. Then, only excitonic states $|0\rangle$ and $|1\rangle$ are taken into account (see Fig. 1). The one-exciton equations can be derived from Eqs. (4) by imposing $\mu_{21}=0$ and $\mu_{10}=1$ as follows:

$$
\begin{aligned}
\rho_{110} & =\frac{\Omega_{0}^{2} / 2}{\frac{\gamma_{10}}{\Gamma_{10}}+\Omega_{0}^{2}}, \\
\frac{\rho_{11 m}}{\Omega_{m}} & =-\frac{\Omega_{0} \Gamma_{10}}{2} \frac{\left(2 \rho_{110}-1\right)\left(2 \Gamma_{10}-i \delta\right)}{\left(\Gamma_{10}-i \delta\right)\left(\gamma_{10}-i \delta\right)+\Gamma_{10}^{2} \Omega_{0}^{2}}, \\
\frac{\Omega_{m}^{\text {in }}}{\Omega_{m}} & =1-\frac{2 \gamma_{R} \Omega_{0} \Gamma_{10}}{\Gamma_{10}-i \delta} \frac{\rho_{11 m}}{\Omega_{m}}-\frac{\gamma_{R} \Gamma_{10}\left(2 \rho_{110}-1\right)}{\Gamma_{10}-i \delta} .
\end{aligned}
$$

The behavior of the sideband field $\Omega_{m}$ for the one-exciton model can be rewritten for clarity as

$$
\frac{\Omega_{m}^{\text {in }}}{\Omega_{m}}=1+\frac{\gamma_{R} \Gamma_{10}}{1+\frac{\Omega_{0}^{2} \Gamma_{10}}{\gamma_{10}}} \frac{\gamma_{10}-\Omega_{0}^{2} \Gamma_{10}-i \delta}{\left(\Gamma_{10}-i \delta\right)\left(\gamma_{10}-i \delta\right)+\Omega_{0}^{2} \Gamma_{10}^{2}},
$$

where two spectral features take place at modulation frequencies $\delta=\gamma_{10}$ and $\delta=\Gamma_{10}$. At modulation frequencies such as $\delta \simeq \gamma_{10} \ll \Gamma_{10}$, the CPO mechanism dominates, which leads to the slow-light propagation regime. From Eq. (6) we calculate an approximation for the fractional shift from $\Omega_{m}^{\text {in }} / \Omega_{m} \simeq(1-i 2 \pi F) / T$. By assuming a small variation of the sideband amplitude, i.e., $T \simeq 1$, we obtain the following well-known expression of CPO-based slow-light delay for the so-called undepleted approximation, where the strong control field $\Omega_{0}$ does not vary significantly along the propagation distance [31]

$$
2 \pi F \simeq \frac{2 \gamma_{R} \Omega_{0}^{2} \Gamma_{10}}{1+\Omega_{0}^{2} \Gamma_{10} / \gamma_{10}} \frac{\delta}{\left(\gamma_{10}+\Omega_{0}^{2} \Gamma_{10}\right)^{2}+\delta^{2}} .
$$

In this regime, the maximum fractional delay is achieved at the modulation frequency $\delta_{\text {opt }}=\gamma_{10}+\Omega_{0}^{2} \Gamma_{10}$, close to the population decay rate $\left(\gamma_{10} \simeq 0.022 \mathrm{THz}\right)$. This optimum modulation frequency corresponds to the FWHM of the spectral hole induced by CPO effects in the absorption spectrum, being power broadened [in agreement with Fig. 2(a)], and gives us an idea of the slow-light operating bandwidth. Furthermore, maximum fractional delay is reached at saturation intensity $\left|\Omega_{0}\right|^{2}=\gamma_{10} / \Gamma_{10}$. Then, the maximum fractional delay is roughly governed by the collective superradiant damping $\gamma_{R}$.

At higher modulation frequencies, in particular at frequencies around the linewidth of the atomic transition $\delta \simeq \Gamma_{10} \gg$ $\gamma_{10}$, the population is not able to follow the field oscillation, and CPO is negligible. Thus, the field, which propagates through the nanofilm inside the anomalous dispersion region suffers advancement, which agrees with simulations. By considering linear light-matter interaction, i.e., the intensity field below the saturation value $\Omega_{0}^{2} \ll \gamma_{10} / \Gamma_{10}$, the sideband equation (6) reduces to

$$
\frac{\Omega_{m}^{\text {in }}}{\Omega_{m}}=1+\frac{\gamma_{R} \Gamma_{10}\left(\Gamma_{10}+i \delta\right)}{\Gamma_{10}^{2}+\delta^{2}}=\frac{1}{T} e^{-i 2 \pi F},
$$

and then the fractional advancement results:

$$
2 \pi F \simeq \arctan \left[\frac{-\gamma_{R} \Gamma_{10} \delta}{\left(1+\gamma_{R}\right) \Gamma_{10}^{2}+\delta^{2}}\right] .
$$

The maximum fractional advancement is achieved at the modulation frequency $\delta_{\mathrm{opt}}=\Gamma_{10} \sqrt{1+\gamma_{R}}$, which is close to the dephasing rate, that is, to the linewidth of the transition, but it is also increasing with the collective superradiant damping $\left(\delta_{\mathrm{opt}} \simeq 5.5 \mathrm{THz}\right.$ for $\left.\gamma_{R}=25\right)$. In addition, $2 \pi F A_{\mathrm{opt}} \simeq$ $\arctan \left(-0.5 \gamma_{R} / \sqrt{1+\gamma_{R}}\right)$, which slightly increases with $\gamma_{R}$ in the range of values used in this work. In particular, $F A_{\mathrm{opt}} \simeq$ -0.18 for $\gamma_{R}=25$. These estimations show a clear agreement with simulations [see Figs. 2(a) and 3(a)].

\section{B. Pulse propagation in disordered nanofilms}

We want to address now the situation of interest for telecommunications: pulsed signal propagation in real (disordered) molecular-aggregate nanofilms. Thus, we illuminate the nanofilm with a Gaussian-like pulse with a FWHM: $\Omega=$ $\Omega_{0}+\Omega_{m} \exp [-2 \sqrt{\ln (2)} t / \mathrm{FWHM}]^{2}$. To test the applicability of the proposed device, the attainable delay or advancement and also the distortion $D$ of the output pulses are calculated. The latter is obtained as the ratio of the standard deviation $s$ of the side-band output and input-pulsed intensities:

$$
D=\frac{s\left(\left|\Omega_{m}\right|^{2}\right)}{s\left(\left|\Omega_{m}^{\text {in }}\right|^{2}\right)}
$$

First, in Fig. 4 we study the fractional shift as a function of the initial pulse temporal width and of the input strong field. Results obtained in the absence (presence) of disorder with $G=0(G=2)$ are compared for $\gamma_{v}=2$. In agreement with results of the previous section, one can observe that optimal values for $F A$ and $F D$ appear for different magnitudes of FWHM and $\Omega_{0}^{\text {in }}$. Indeed $F A_{\text {opt }}$ is obtained for low input fields and pulses in the $\mathrm{THz}$ range, while $F D_{\text {opt }}$ arises for input fields of the order of the saturation intensity and pulses with FWHM $\sim 10 \mathrm{GHz}$. Such a difference in the time scales is directly related to the optical mechanisms playing a role in every process. Thus, the fast-light time scale is related to the natural resonance width of the system $\left(\sim \Gamma_{10}\right)$, while the slow light is dominated by $\mathrm{CPO}$ and then by the spontaneous decay rate of the aggregate $\left(\sim \gamma_{10}\right)$. This analysis is robust under the 

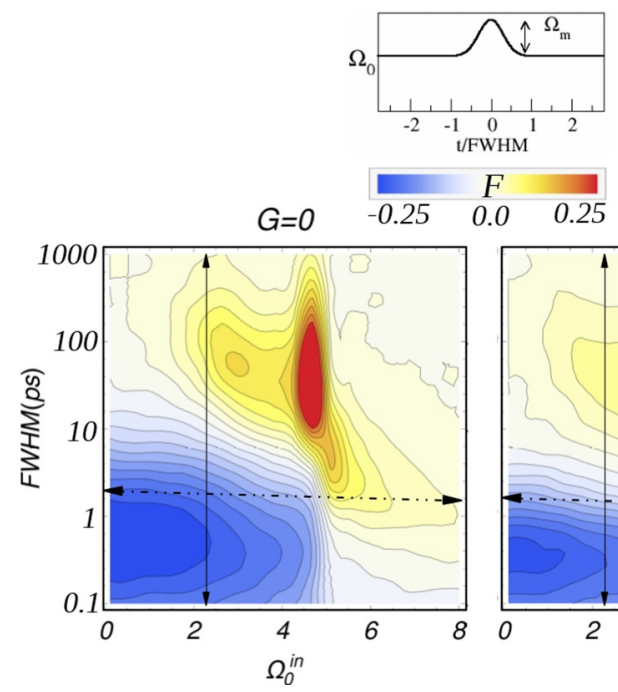

\section{$G=2$}

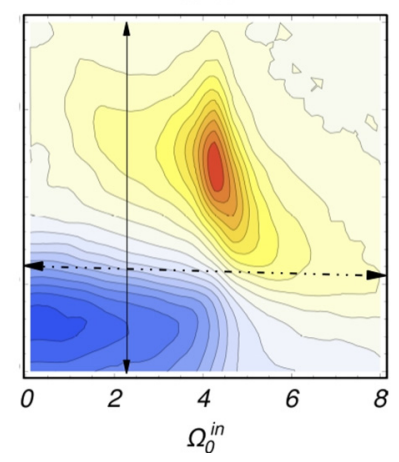

FIG. 4. Contour plot of the fractional shift $(F)$ as a function of the initial pulse temporal width (FWHM) and of the input strong field $\left(\Omega_{0}^{\text {in }}\right)$ for $\gamma_{v}=2$. Results in absence of disorder $G=0$ (left panel) are compared with those obtained in the presence of disorder with $G=2$ (right panel). The cut along the solid (dashed) arrow is analyzed in Fig. 5 (Fig. 6). Top panel presents a schematic plot of the considered Gaussian-like pulses.

inclusion of disorder in the system although the achievable fractional shifts are slightly reduced in comparison to the homogeneous case, as shown in the right panel of Fig. 4. Furthermore, as we will discuss below, Fig. 4 shows a potential transition from fast light to slow light by tuning the amplitude of the input strong field (indicated by the horizontal dashed arrow in both panels). Figure 5 shows the fractional shift, transmission, and distortion of pulsed signals, as a function of its FWHM for different magnitudes of disorder. In Fig. 5(a), similar to Fig. 2(a), we obtain fast- and slow-light performace for a particular range of pulse widths in the GHz-THz bandwidth. Pulses with a GHz bandwidth will be inside the CPO hole in the absorption spectrum, then all the spectral components will be delayed. However, when the pulse gets spectrally wider, to the $\mathrm{THz}$ region, most of the spectral components will observe the anomalous dispersion region of the natural resonance and then propagate in a superluminal regime. Disorder decreases the attainable $F A$ and $F D$ but still one
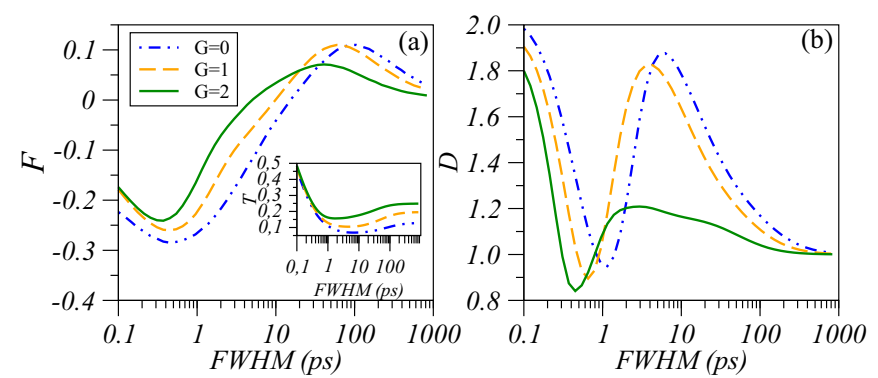

FIG. 5. (a) Fractional shift, (b) distortion, and (inset) transmittance for pulsed input signals against the initial pulse temporal width (FWHM) for the ratio $\gamma_{v}=2$ and $\Omega_{0}^{\text {in }}=\Omega_{0}^{\text {sat }} / 2$. Different magnitudes of disorder $G$ are considered.

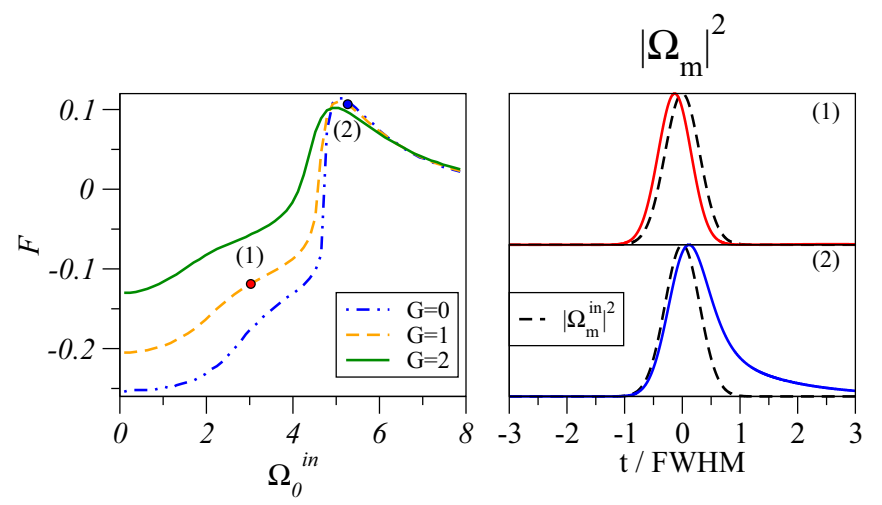

FIG. 6. Tunable fractional shift transmittance for a pulsed input signal as a function of the input strong field $\Omega_{0}^{\text {in }}$ for a pulse of $\mathrm{FWHM}=2$ ps. Different magnitudes of disorder $G$ and $\gamma_{v}=2$ are considered. Output and input pulses for fields (1) and (2) are shown in the right panel.

could tune the device to obtain a fractional shift of $F=$ \pm 0.1 . Interestingly distortions remain within useful values for telecommunications $D<2$, see Fig. 5(b). Last, we propose an additional functionality of the molecular-aggregate nanofilm to control the group velocity of a particular pulsed signal as a function of the incident field. Figure 6 pictures the transition from fast- to slow-light performance for a pulse of FWHM $=2$ ps by increasing the external field. As it is shown, the maximum attainable $F A$ is obtained for lower input peak fields corresponding to a time advancement of -0.6 to -0.25 ps for $G=0$ and $G=2$, respectively. Although the maximum advancement is affected by disorder, the maximum delay is almost constant for the analyzed values of $G$, giving a time delay of $\sim 0.2$ ps for this input bandwidth.

\section{EXPERIMENTAL CONDITIONS}

Let us first emphasize the peculiar role of $J$-aggregates for the proposed nanometric slow- and fast-light tunable device. One of the best known optical properties of $J$-aggregates is the absorption band narrowing due to aggregation [32]. This effect is directly related to a reduction of the magnitude $\Gamma_{10}$ that approximately accounts for the absorption bandwidth. Regarding slow- and fast-light propagation, in Sec. III A we have concluded that the maximum delay or advancement increases with the collective superradiant damping $\gamma_{R}$. Therefore, it is clear that if aggregation is destroyed, an increase of $\Gamma_{10}$ would lead to a strong reduction of the achieved delay or advancement. In addition, the particular modulation frequencies for which these optimum shifts will arise also depend on the aggregation length. For slow-light propagation it was demonstrated that $\delta_{\mathrm{opt}} \sim \gamma_{10}^{N}=N \gamma_{10}^{1}$ and thus is increased due to aggregation, while for fast-light propagation the optimum modulation frequency $\delta_{\text {opt }} \sim \Gamma_{10}$ would be reduced. As a result if aggregation were not present, the bandwidth region affected by efficient slow light would be shifted apart from that for fast light. Therefore, the particular properties of $J$-aggregates not only give rise to a clear improvement of the achieved delay or advancement but also allow a much more 
efficient tunability of the system from slow-light to fast-light performance.

After the previous analysis, it is essential to check the feasibility of a fast- and slow-light experiment on $J$-aggregates. First, if we compare the current four-level model with its simplified version where two-exciton dynamics is neglected [3], the values of $\gamma_{R}$ needed to achieve large fractional shifts increase up to $\gamma_{R} \simeq 30$. Recalling the expression of $\gamma_{R}=\mu_{0}|d|^{2} N_{0} c \omega L / 2 \hbar \Gamma_{10}$, these values of the superradiant constant corresponds to an aggregates density $N_{0} \sim 5 \times$ $10^{23} \mathrm{~m}^{-3}$ for a thickness $L \sim \lambda / 10$ in order to keep valid the ultrathin film approximation used along the article. Both values can be achieved by vertical spin-coating [33]. Moreover, our calculations involve fast pulses with bandwidths in the range of $0.01-1 \mathrm{THz}$ for $\lambda_{0}=567 \mathrm{~nm}$, available by current optical parametric amplifiers. A harder condition is imposed, however, by the optical damage threshold of a $J$-aggregate nanofilm. Although this magnitude must be eventually measured in experiments, we can make a sensible estimation by looking at a representative value of $\Omega$, namely, the Rabi frequency inside the aggregate nanofilm. For example, by considering $\Omega_{\text {in }} \sim 6$ for $\gamma_{R}=30$, then $\Omega \sim 2$ [see inset of Fig. 2(b)], and an incident intensity of $\lesssim 10^{18}$ photons $/ \mathrm{m}^{2}$ per pulse is obtained for 70-ps-long pulses. This value is even an order of magnitude lower inside the nanofilm, so our device operates below the damage threshold measured in some experiments (see, for instance, Ref. [34]). Notice that these values are calculated by pulses affected by the slow-light regime. Fast light is achieved for shorter pulses, with a temporal width close to $1 \mathrm{ps}$, and for lower values of the electric field. Therefore, the fast-light regime is clearly less critical than the previous estimation and no photobleaching is expected.

\section{CONCLUSIONS}

In summary, slow- and fast-light tunability has been proven for $J$-aggregate nanofilms for pulses with bandwidths close to the $\mathrm{THz}$ range, due to the coherent response of the aggregate. Such tunability can be reached by changing the input pulse power or its temporal width. Moreover, both slow- and fastlight regimes can be reached in the presence of disorder, a fundamental condition for its applicability in technological solutions. We used a four-level model describing one- and two-exciton states and the annihilation of the exciton-exciton state by means of a set of electronic-vibrational levels. A simple analytical approach of this model was carried out to clearly point out the mechanisms involved in the slowand fast-light regimes, that is, CPO-based slow-light and fast-light propagation in the anomalous dispersion region of the atomic resonance. The nanometric size and the removal of the external pump dependence to obtain fast light make molecular-aggregate nanofilms very promising candidates for integrated all-optical solutions to light-velocity control.

\section{ACKNOWLEDGMENT}

This research has been supported by Ministerio de Economa y Competitividad (MINECO) (MAT2013-46308, MAT2016-75955, FIS2013-41709-P, MAT2017-8 3111-R).
[1] J. Mørk, R. Kjær, M. van der Poel, and K. Yvind, Opt. Express 13, 8136 (2005).

[2] K.-H. Kim, A. Husakou, and J. Herrmann, Opt. Express 20, 25790 (2012).

[3] E. Cabrera-Granado, E. Díaz, and O. G. Calderón, Phys. Rev. Lett. 107, 013901 (2011).

[4] E. Díaz, G. C. Martínez-Calzada, E. Cabrera-Granado, and O. G. Calderón, Opt. Lett. 41, 2569 (2016).

[5] T. Kobayashi, J-aggregates (World Scientific, Singapore, 1996).

[6] F. C. Spano and J. Knoester, Advances in Magnetic and Optical Resonance (Academic, New York, 1994).

[7] R. W. Boyd and Z. Shi, Slow and Fast Light (Wiley, New York, 2015), pp. 363-385.

[8] L. J. Wang, A. Kuzmich, and A. Dogariu, Nature 406, 277 (2000).

[9] S. Chu and S. Wong, Phys. Rev. Lett. 48, 738 (1982).

[10] H. Tanaka, H. Niwa, K. Hayami, S. Furue, K. Nakayama, T. Kohmoto, M. Kunitomo, and Y. Fukuda, Phys. Rev. A 68, 053801 (2003).

[11] J. Keaveney, I. G. Hughes, A. Sargsyan, D. Sarkisyan, and C. S. Adams, Phys. Rev. Lett. 109, 233001 (2012).

[12] S. Jennewein, Y. R. P. Sortais, J.-J. Greffet, and A. Browaeys, Phys. Rev. A 94, 053828 (2016).

[13] H. Glaeske, V. A. Malyshev, and K. H. Feller, J. Chem. Phys. 114, 1966 (2001).
[14] H. Glaeske, V. A. Malyshev, and K.-H. Feller, Phys. Rev. A 65, 033821 (2002).

[15] J. A. Klugkist, V. A. Malyshev, and J. Knoester, J. Chem. Phys. 128, 084706 (2008).

[16] J. A. Klugkist, Mechanisms for Photonic Switching in Systems of Strongly Interacting Dipoles (Zernike Institute $\mathrm{Ph} . \mathrm{D}$. thesis series 2008-4, Groningen, The Netherlands, 2008), p. 86.

[17] H. Fidder, J. Knoester, and D. A. Wiersma, J. Chem. Phys. 98 , 6564 (1993).

[18] E. C. Jarque and V. A. Malyshev, J. Chem. Phys. 115, 4275 (2001).

[19] A. I. Zaitsev, V. A. Malyshev, and E. D. Trifonov, Sov. Phys. JETP 57, 275 (1983).

[20] M. G. Benedict, A. M. Ermolaev, V. A. Malyshev, I. V. Sokolov, and E. D. Trifonov, Super-radiance: Multiatomic Coherent Emission (Institute of Physics, Philadelphia, 1996).

[21] G. Piredda and R. Boyd, J. Eur. Opt. Soc. Rapid Pub. 2 (2007).

[22] D. J. Harter and R. W. Boyd, J. Quantum Electron. 16, 1126 (1980).

[23] S. DeBoer and D. A. Wiersma, Chem. Phys. Lett. 165, 45 (1990).

[24] H. Fidder, J. Knoester, and D. A. Wiersma, Chem. Phys. Lett. 171, 529 (1990).

[25] K. Minoshima, M. Taiji, K. Misawa, and T. Kobayashi, Chem. Phys. Lett. 218, 67 (1994)

[26] H. Stiel, S. Daehne, and K. Teuchner, J. Lumin. 39, 351 (1988). 
[27] V. V. Gusev, Adv. Mater. Opt. Electron. 1, 235 (1992).

[28] V. Malyshev and P. Moreno, Phys. Rev. A 53, 416 (1996).

[29] V. A. Malyshev, H. Glaeske, and K. H. Feller, J. Chem. Phys. 113, 1170 (2000).

[30] R. Boyd, Nonlinear Optics (Academic, New York, 2008).

[31] F. Arrieta-Yáñez, O. G. Calderón, and S. Melle, J. Opt. 12, 104002 (2010).
[32] M. Oda, Y. Obara, K. Saito, K. Higashi, and T. Tani, Phys. Proc. 3, 1615 (2010).

[33] K. Misawa, H. Ono, K. Minoshima, and T. Kobayashi, Appl. Phys. Lett. 63, 577 (1993).

[34] R. V. Markov, Z. M. Ivanova, A. I. Plekhanov, N. A. Orlova, and V. V. Shelkovnikov, Quantum Electron. 31, 1063 (2001). 